\section{Enchondroma of the Lunate}

\author{
Chul Han Kim \\ Department of Plastic and Reconstructive Surgery, \\ Soonchunhyang University College of Medicine, Seoul, Korea
}

\section{Correspondence: Chul Han Kim}

Department of Plastic and Reconstructive Surgery, Soonchunhyang University College of Medicine, 59 Daesagwan-ro, Yongsan-gu, Seoul 140-743, Korea Tel: +82-2-709-9283, Fax: +82-2-796-3543

E-mail: kchann@hanmail.net

This work was supported in part by the Soonchunhyang University Research Fund. No potential conflict of interest relevant to this article was reported.

Received: 4 May 2012 • Revised: 11 May 2012 • Accepted: 13 May 2012 pISSN: 2234-6163・ elSSN: 2234-6171

http://dx.doi.org/10.5999/aps.2012.39.6.678 • Arch Plast Surg 2012;39:678-680

Copyright @ 2012 The Korean Society of Plastic and Reconstructive Surgeons This is an Open Access article distributed under the terms of the Creative Commons Attribution Non-Commercial License (http://creativecommons.org/licenses/by-nc/3.0/) which permits unrestricted non-commercial use, distribution, and reproduction in any medium, provided the original work is properly cited.

Enchondroma is a benign, intramedullary cartilaginous bone tumor that is usually found in the small tubular bones [1]. Enchondroma is usually revealed incidentally on radiography taken for other causes or as a pathologic fracture. X-ray radiography of enchon- droma shows a round, transparent osteolytic bony change [2]. It is generally asymptomatic, but may expand [3]. Although enlargement of enchondroma with cortical thinning and protrusion can occur in the advanced phase, the cortical margin of the involved bone is spared, and there is no invasion into the surrounding tissue [2].

Enchondroma usually occurs in the phalanges and metacarpals [1]. However, it is rarely found in carpal bone [2-4]. We report a case with an enchondroma occurring in the lunate bone of the carpus, which was associated with dorsal wrist pain.

A 40-year-old right-handed woman complained of intermittent right wrist pain for the previous 8 months (Fig. 1). The symptoms worsened after prolonged usage of the hand. She had no history of previous trauma. Eight months earlier, she had experienced painful swelling in the right wrist, which was diagnosed as tendonitis and treated with a non-steroidal antiinflammatory drug at a local clinic. Physical examination revealed dorsal wrist tenderness, and wrist movements were not restricted. Laboratory findings were within normal limits. An X-ray of the wrist showed a well-demarcated, rounded radiolucent area in the lunate. There were no fractures or other lesions in the bones of the hand. Magnetic resonance imaging (MRI) demonstrated that T1-weighted images showed hypointense and $\mathrm{T} 2$-weighted images hyperintense signal differences from the normal bony margin of the lunate (Fig. 2).

Surgery was performed with a dorsal approach to the lunate bone. After careful dissection, the lunate was exposed. The cortex of the lunate was very thin and a small burr was used to open a cortical window. The inner contents were meticulously curetted and

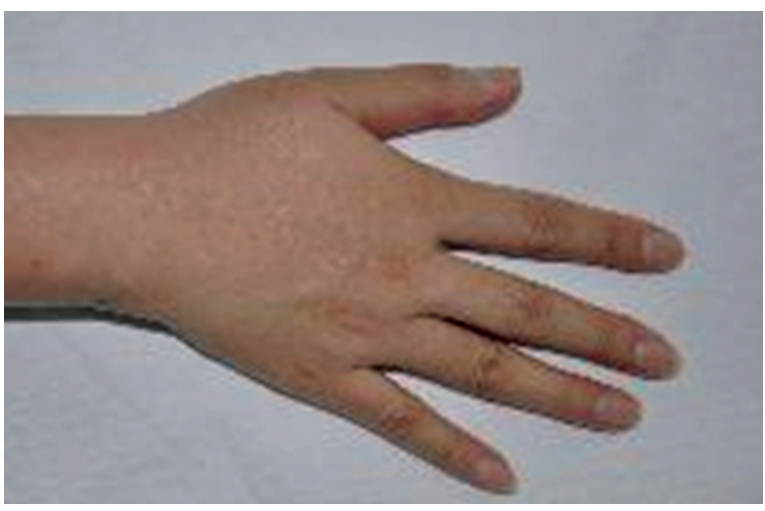

Fig. 1.

A 40-year-old woman presented with pain in the dorsal aspect of the right wrist.

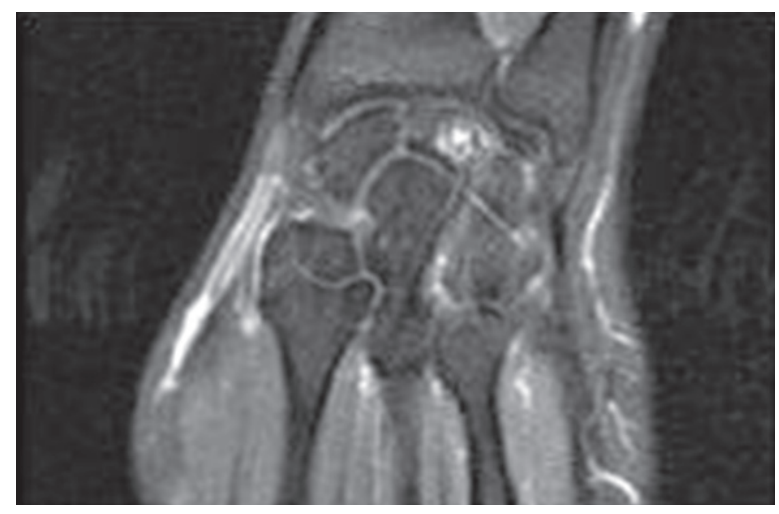

Fig. 2.

A preoperative T2-weighted magnetic resonance imaging image revealed a high signal intensity in the lunate. 


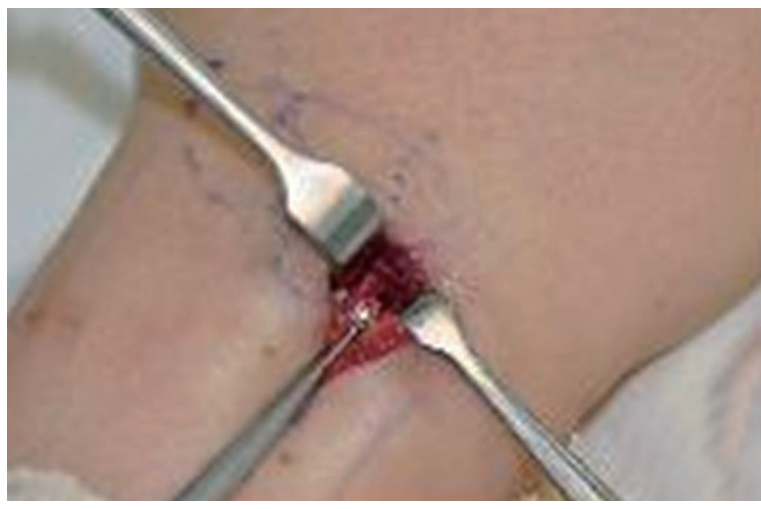

Fig. 3.

Intraoperative findings. The lesion was meticulously curetted and biopsied after the exposure of the cortex by a small burr.

biopsied (Fig. 3). Histopathology of the specimen revealed the typical lobular pattern of enchondroma. The cells were relatively small with no evidence of nuclear atypia or double nucleated chondrocytes (Fig. 4). The diagnosis of enchondroma was made. The defect cavity of the lunate was grafted with corticocancellous bone taken from the iliac crest to maintain bone integrity. The capsular and ligamentous complex were then repaired. The wrist was immobilized for 3 weeks by a short arm splint. The postoperative course was free of any complication. Within six months, she experienced considerable improvement of symptoms, and she returned to work without difficulty. X-rays showed complete incorporation of the graft, and no sign of recurrence.

Enchondromas involving the hand have a predilection for the ulnar digits and the preferred locations of enchondromas are the distal metacarpals and proximal phalanges [1]. However, enchondroma in the carpal bone is rare [2-4]. In addition, carpal enchon- dromas have mostly been reported in the scaphoid $[1,3,4]$.

Enchondroma is generally asymptomatic, but it may cause growth of the associated bone. It causes endosteal resorption and cortical thinning, and thus may predispose the involved bone to pathologic fracture $[3,4]$. Many cases of scaphoid enchondroma have been diagnosed after a pathologic fracture [4]. However, our case of lunate enchondroma presented as dorsal wrist pain without fracture. We concluded that enchondroma of the lunate, such as scaphoid enchondroma, could lead to pathologic fracture and subsequent carpal collapse.

Although the plain X-ray findings are characteristic in diagnosis of enchondroma, diagnosis of enchon-

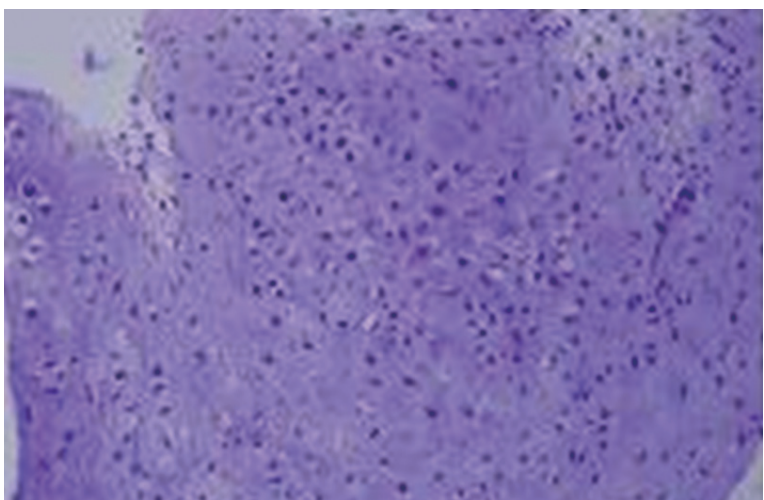

Fig. 4.

Histologic findings demonstrated the typical lobular pattern of enchondroma with a moderate amount of cellularity and relatively small cells with no evidence of nuclear atypia or double nucleated chondrocytes $(H \& E, \times 200)$.

droma of the carpal bone may require further radiodiagnostic imaging such as computed tomography (CT) or MRI [3]. MRI is particularly helpful in identifying enchondroma, which demonstrates low signal intensity on T1-weighted images and high signal intensity on T2-weighted images [3].

Treatment was aimed at the complete curettage of the lesion, prevention of recurrence, and reconstruction of the bone defect with a graft procedure [4]. Reported recurrence rates of enchondromas vary, and inadequate curettage is thought to be the main cause of recurrence [2]. Autogenous bone graft is considered inevitable for carpal bone disorders such as enchondroma [4]. In scaphoid enchondromas, surgical enucleation with autogenous bone graft was reported as a definitive therapy with few complications [2]. Our case of lunate enchondroma was also successfully treated by curettage and bone graft from the iliac bone. We believe that performing complete curettage and bone graft minimizes morbidity and preserves wrist function.

It may be controversial whether surgical treatment of enchondroma suspected on the basis of clinical and radiologic findings is necessary. A solitary painless enchondroma may be observed. However, if the tumor is painful or if radiographic findings, including MRI or CT, are inconclusive, the diagnosis should be confirmed by biopsy, which must be analyzed carefully for malignancy. Enchondroma has been reported to be difficult to differentiate from low grade chondrosarcoma by radiology [5]. Hence, we believe that surgical removal of enchondroma is necessary because it will establish an accurate histopathologic diagnosis, 
free the patient of clinical symptoms, and strengthen the mechanical stability of the involved bone.

Malignant degeneration of benign enchondroma to chondrosarcoma has been reported [5]. It was characterized by a sudden increase in size with a soft tissue mass and pain in a previously asymptomatic lesion. Radiographic evidence may show a less confined osteolytic lesion and appearances of cortical destruction with soft tissue extension [5].

This case of enchondroma involved the carpal lunate bone. For proper diagnosis, conventional X-ray examination and MRI were performed. Histopathologic study should be obligatory because of possible malignancy. The treatment of choice is the complete removal of the lesion by curettage and bone graft. Although enchondroma is usually a painless lesion and asymptomatic enchondroma in the hand is not unusual, carpal enchondroma of the lunate should be recognized as a possible cause of dorsal wrist pain despite the rarity of its occurrence.

\section{References}

1. Gaulke R. The distribution of solitary enchondromata at the hand. J Hand Surg Br 2002;27:444-5.

2. Takigawa K. Chondroma of the bones of the hand. A review of 110 cases. J Bone Joint Surg Am 1971;53: 1591-600.

3. Takka S, Poyraz A. Enchondroma of the scaphoid bone. Arch Orthop Trauma Surg 2002;122:369-70.

4. Masada K, Fujiwara K, Yoshikawa H, et al. Chondroma of the scaphoid. J Bone Joint Surg Br 1989;71:705.

5. Nelson DL, Abdul-Karim FW, Carter JR, et al. Chondrosarcoma of small bones of the hand arising from enchondroma. J Hand Surg Am 1990;15:655-9. 